\title{
Linker histones: history and current perspectives
}

\author{
C Crane-Robinson, Biophysics Laboratories, School of Biology, University of \\ Portsmouth, P01 2DT, UK.
}

\begin{abstract}
Although the overall structure of the fifth histone (linker histone, H1) is understood, its location on the nucleosome is only partially defined. Whilst it is clear that H1 helps condense the chromatin fibre, precisely how this is achieved remains to be determined. $\mathrm{H} 1$ is not a general gene repressor in that although it must be displaced from transcription start sites for activity to occur, there is only partial loss along the body of genes. How the deposition and removal of $\mathrm{H} 1$ occurs is in particular need of further study. Linker histones are highly abundant nuclear proteins about which we know too little.
\end{abstract}

The functional role of the fifth histone (H1, for short in what follows) has proven the most difficult to understand. While the structure of the octameric $147 \mathrm{bp}$ core particle [1] rapidly led to an enhanced appreciation of core histone function, in particular regarding the roles of their different structural domains, no equivalent structure has been obtained for an H1-containing nucleosome. The main reason is that reconstitution of the very basic $\mathrm{H} 1$ molecule with an extended core particle of high net negative charge is subject to artifactual non-specific interactions and, furthermore, there is no obvious functional assay for correct binding. Additionally, chromatin-bound $\mathrm{H} 1$ is known to rapidly exchange [2,3] (in vivo, at least) so the conditions for generating a stable in vitro H1-containing chromatosome are not yet defined. Even more uncertain are methods for generating H1-containing higher order chromatin structures, such as the so-called $30 \mathrm{~nm}$ supercoil (30 $\mathrm{nm}$ fibre). So the primary issues in need of resolution are to understand precisely how $\mathrm{H} 1$ is bound to the nucleosome and how this helps the formation and stabilisation of the $30 \mathrm{~nm}$ fibre. Only then will we be able to fully comprehend how chromatin structures are controlled to facilitate the various transactions to which the DNA is subject.

Structure of linker histones. It was early appreciated that H1 plays a role in condensing chromatin and studies of its free solution structure gave hints as to how 
it achieves this. Limited proteolysis showed that most $\mathrm{H} 1$ species consist of 3

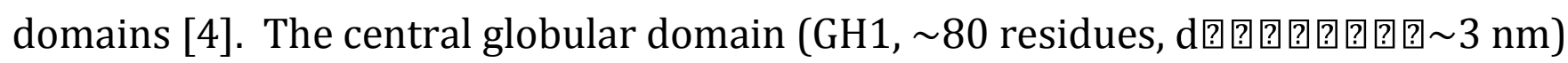
is the only folded element in the $\sim 200$ amino acid protein in free solution. It adopts the winged helix fold [5]. The short N-terminal domain (NTD, 35 residues) is very basic but only in its second half: in contrast, the N-terminal part is somewhat apolar, even acidic. The NTD is disordered in free solution and does not play an obvious role in chromatin condensation [6] but is subject to post-translational modifications (PTMs), so may play a regulatory/signalling role in $\mathrm{H} 1$ function. Linker histones specific for condensed chromatin typically have a shorter NTD but in oocyte specific H1s it is often longer. The C-terminal domain (CTD) is also disordered in free solution but is much longer: $\sim 100$ residues in the canonical mammalian species but $>200$ residues in some oocyte-specific linker histones. Since the CTD is very lysine rich it is assumed to play the main role in condensing the nucleosomal fibre, a function modulated by phosphorylation at multiple sites, i.e. the addition of negative charges [6-9]. An important but difficult question to which a definitive answer is needed is the extent to which the NTD and CTD adopt defined secondary structures when bound into the chromatin fibre $[10,11]$.

H1s and their variants The canonical mammalian replication-dependent (RD) H1 subtype genes (H1.1 to H1.5 and the testis-specific H1t in humans) are encoded within multiple histone clusters together with those of the core histones, synthesised only during S-phase [12]. So despite the substantial structural and functional distinctions between the core and linker histones, the H1s are an intrinsic component of the chromatin of higher eukaryotes - quite different from the situation in yeast (Saccharomyces cerevisiae) where there are only two copies of each of the core histone genes $[13,14]$ and the presumed linker histones look very different from mammalian H1s.

As with the core histones $\mathrm{H} 2 \mathrm{~A}$ and $\mathrm{H} 3$, in mammalian cells there are also a number of replacement (variant) H1s, synthesised throughout the cell cycle from genes that sometimes contain introns and generate polyA+ mRNAs. Variant H1s play 
very specific roles: for example, H5 from the nucleated erythrocytes of birds, and its mammalian homologue H1.0, are both a feature of highly compacted chromatin, probably playing a condensing role by including multiple arginines (largely absent from the canonical mammalian $\mathrm{H} 1$ subtypes). Oocyte-specific variants, for example xenopus B4 and mammalian H1oo, have CTDs with a substantial admixture of acidic residues (E and D) - amino acids totally absent from the CTDs of canonical mammalian H1s.

H1 PTMs Extensive phosphorylation of serine and threonine in the NTD and especially the CTD of canonical H1s was documented early and linked to chromatin condensation at mitosis [15]. ADP-ribosylation of the NTD was also early recognised [16] and might be thought of a component of the histone code but H1s were initially not thought to be acetylated or methylated at lysines. Mass spectrometry has shown not to be the case [17]: for example, K26 of human H1.4 in the NTD can be methylated and the adjacent residue S27 phosphorylated. The same H1 subtype can be acetylated at K34, a residue on the border of the NTD and globular domains. Other modifications have been noted within GH1, notable citrullination of R54 in ESCs [18], a conserved residue within DNA binding Site II (see below).

Histone $\mathrm{H1}$ in the $30 \mathrm{~nm}$ fibre. The first and much vexed question was the location of GH1 on the nucleosome. On the assumption that each nucleosome carries a single $\mathrm{H} 1$ molecule [19] and the observation that GH1 alone is able to protect an extra $10 \mathrm{bp}$ at each end of the core particle, giving the $167 \mathrm{bp}$ chromatosome, it was proposed that GH1 binds symmetrically to the DNA on the dyad axis, making contact with both exiting duplexes and the central gyre: a 3-contact model [4]. Although a symmetrical location was supported by DNaseI footprinting experiments on native chromatin [20], it provoked the question as to what determines the orientation of the linker histone on the nucleosome, so the model was widely challenged both experimentally and by molecular dynamics calculations of preferred orientations [21-23]. General agreement emerged that GH1/5 carries just two DNA binding sites [24,25]: Site I on 
Helix 3 and Site II on the Loop between Helix 1 and Helix 2. This led to proposed interactions being with the central DNA gyre and just one of the exiting duplexes, i.e. a slightly off-axis binding site - as found in a DNA-GH5 crosslinking experiment [26]. Such locations did not however offer any explanation for symmetrical MNase protection of $2 \times 10 \mathrm{bp}$ beyond the core particle.

To extend knowledge of how H1 (in particular GH1/5) is bound to the nucleosome, i.e. define where it sits in the compacted state, neutron scattering contrast measurements were made of the $30 \mathrm{~nm}$ fibre containing native and deuterated $\mathrm{H} 1$ [27]. Plots of the square of the radius of gyration against the inverse contrast made it clear that the increased contrast in the deuterated sample must come from internally located $\mathrm{H} 1$. Whatever the precise model of $\mathrm{H} 1$ binding, this indicated that the DNA exit points of the nucleosomes must be on the inside of the fibre. Although this result did not lead to a description of nucleosome connectivity within the fibre (continuous solenoid vis-à-vis cross-linker models), it excluded all models with outward facing nucleosomes.

Appreciation of a possible third DNA binding site on GH1/5 [28,29,22,30] led to continuing interest in a symmetrical location and this was supported by hydroxyl radical footprinting studies (giving higher resolution than DNaseI [20]) that showed a clear shadow from GH1 protection right on the dyad [31]. As the latter studies were conducted on reconstituted short oligo-nucleosomes, it was also possible to investigate the linker DNA. Strikingly, this showed protection by $\mathrm{H} 1$ of $\sim 50$ bp (170 A) of linker with a alternating period of $10 \mathrm{bp}$, implying extended shielding from one side - presumed to result from the CTD as deletion of the 35-residue NTD did not affect the protection. The success of these footprinting experiments [31] may well have been due to the use of a chaperone (NAP1) for loading the H1s onto the chromatin template, an approach full of perspective. 
Further evidence for the location of GH1 came from a cryo-EM study [32], at $\sim 11 \AA$ resolution, of crosslinked 12-mer arrays of H1.4-containing nucleosomes, which appear as three tetranucleosomal units stacked to form a zig-zag 2-start helix, each unit having fully extended DNA linkers. Fitting the crystal structure of the $167 \mathrm{bp}$ repeat tetranucleosome (that lacks H1) [33] to the averaged cryo-images allowed an assessment of the location of H1. Well-defined density was observed between the exiting DNA duplexes: fitting this to the crystal structure of free GH5 [5] led to a 3contact model for the globular domain in which Site III, (comprising the two $\beta$-strands of Loop 3), is in contact with the central DNA gyre of the nucleosome and Sites I and Site II make contact with the two exiting DNA duplexes. This location put GH1 somewhat asymmetrically placed, the Site III contact being about one quarter of a turn $(\sim 8 \AA)$ from the dyad axis.

Even more recently, however, a crystal structure of a reconstituted GH5containing $167 \mathrm{bp}$ chromatosome at $3.5 \AA$ resolution was published [34] showing the H5 globular domain located essentially symmetrically on the dyad axis, with 3 contact regions: Loop 3 (i.e. Site III, e.g. S90 and V87) is in contact with the central DNA gyre (major groove); Loop 1 (e.g. R42) is in contact with the minor groove of one exiting DNA duplex and Helix 3 (e.g. Q67) is edge on into the minor groove of the other exiting duplex, in addition to several K/R-phosphate contacts. This is essentially the symmetrical model originally proposed in our 1980 article [4] but this does not imply that all $\mathrm{H} 1$ globular domains are necessarily placed so symmetrically. The authors of the GH5 chromatosome structure themselves previously used several other physical techniques, i.e. not crystallography, to study the nucleosomal binding of the globular domain of Drosophila H1 [35] and concluded that binding is asymmetric: contact is made with the central DNA gyre and with one exiting duplex. This conclusion was reinforced in their latest publication [34] in which they compared the sedimentation of a 12-nucleosome array carrying GH5 or Drosophila GH1: the GH5 array was seen to be substantially more compact, implying that the binding mode of linker histones is variable and can influence the higher order folding of chromatin. This is unquestionably an attractive hypothesis: the existence of 
multiple subtypes and variants of the linker histones are clearly related to disparate functions that must surely imply variations of structure in the resulting chromatin.

A primary aim of structural studies of $\mathrm{H} 1$ in the context of the fibre has always been to understand how it compacts the nucleosomal beads-on-a-string structure. Since H1-containing nucleosomal arrays adopt a rigid zig-zag structure that compacts to form the $30 \mathrm{~nm}$ fibre [36-39], the first assumption was that GH1 directs the exit angles of the DNA from the nucleosome and the basic CTD covers the linker DNA, the resulting charge reduction permitting folding and compaction. Such a general principle of $\mathrm{H} 1$ involvement in folding could hold for all models of nucleosome connectivity within the fibre. However, such a mechanism suggests that without H1 the $30 \mathrm{~nm}$ fibre might not form at all - but this is not the case: the presence of divalent cations is sufficient to compact an H1-depleted regular nucleosomal array [40]. A possible explanation for this is seen in AFM observations that the DNA duplexes exiting from nucleosomes lacking $\mathrm{H} 1$ cross at right angles, provided 4-10 $\mathrm{mM} \mathrm{Mg++}$ is present [41]. Thus Mg++ could help order the DNA architecture within the fibre but how such divalent cations might be sufficient to counteract repulsions between linker DNAs on the inside of the fibre remains an open question.

The precise geometry of the nucleosomes and the trajectory of the linker DNA in the folded $30 \mathrm{~nm}$ chromatin fibre has been the subject of much study and dispute. The 167 bp repeat tetranucleosome, lacking H1, [33], as well as the recent cryo-EM structure [32] showed a stacked arrangement with fully extended DNA linkers. This has lent support to models in which the linker DNA between adjacent nucleosomes criss-crosses the fibre and several arrangements are physically possible [42]. In vitro studies of longer fibres typically use arrays of a core octamer-positioning sequence, such as the Widom 601 sequence [43] - which allows for variations in the spacing, i.e. DNA linker length - reconstituting with core histones and H1. The resulting 'fibre' has been observed by both negative staining and cryo-EM and a helix of nucleosomes proposed, the dimensions of which depend on the NRL used in the constructs [44- 
47]. The EM images imply that the structure is not particularly rigid and thus not perfectly regular, even over short distances; additionally, the compaction of the nucleosomal fibre obscures the path taken by the centrally located linker DNA. Nevertheless, the Rhodes laboratory propose that the inter-digitated helix that best fits their data is a 1-start solenoid with continuously wound linker DNA [45]. The recent cryo-EM study [32] presents a clear cross-linker structure, moreover not just for a $167 \mathrm{bp}$ NRL but also for the more typical $187 \mathrm{bp}$.

The conflict between bent linker and straight, cross-linker, models is however by no means fully resolved, not least because most observations to date have been made on chromatin fragments on surfaces or in the solid state. High resolution biochemical experiments in solution would be helpful to define the path of the internucleosomal linker DNA, i.e. define the internal architecture of the fibre. It is worth noting here that as well as on EM grids there is good solution evidence for in vitro 30 nm fibres [48], to complement observations of the fibre in the highly condensed chromatin of sperm and nucleated erythrocytes [47]. However, no dominant scattering peaks corresponding to this distance were obtained from active somatic cells, either in interphase or even at mitosis [49-51].

Rapid dynamics of H1 FRAP/FLIP experiments have shown that a large proportion of the $\mathrm{H} 1$ in mammalian nuclei exchanges rapidly between sites on euchromatin with an average residence time of $\sim 4 \mathrm{~min}$, a process accelerated after treatment of cells with TSA to boost histone acetylation levels [2,3]. This in in sharp contrast to the core histones for which essentially no exchange is observed over the same time period. The residues on the surface of GH1 responsible for $\mathrm{H} 1.0$ binding to chromatin have been mapped in vivo by FRAP, measuring the exchange rates of a large set of GH1.0 point mutants [23]. Two clusters of basic residues were highlighted for which mutation to alanine led to much increased dissociation rates, indicating that these represent nucleosomal binding sites - in agreement with the two binding sites previously defined, Site I and Site II. This led the authors to favour an off-axis model in which GH1.0 is in contact with the central DNA gyre and just one exiting duplex. 
Additionally, mutations of $A$ and $S$ to $D$ in the $\beta$-loop wing element of the fold also led to much increased dissociation rates [23], perhaps also indicative of a third binding site, that would be consistent with symmetric on-axis 3-site binding models. The importance of Site II was seen from a R42A mutation that led to a 3-fold increase in the dissociation of GH1.0 [23] and this was strikingly confirmed by the recent demonstration that this residue is subject to citrullination by PADI-4 (conversion of arginine to citrulline) in pluripotent mouse ESCs, leading to displacement of the H1 from chromatin [18]. This modification must be a factor contributing to the rapid mobility of H1s and the consequent 'open chromatin' characteristic of mESCs [52].

Such dynamic binding of $\mathrm{H} 1[2,3,23]$ seems inconsistent with the ubiquitous presence in cells of a tightly wrapped $30 \mathrm{~nm}$ fibre having internal $\mathrm{H} 1$, so the short residence times observed can be regarded as indirect evidence that the $30 \mathrm{~nm}$ fibre is itself dynamic in nature, at least in vivo, which might also explain the failure to detect it in the nuclei of most cell types [49-51].

The functionality of $\mathbf{H 1}$ in chromatin. Although the stoichiometry of $\mathrm{H} 1$ in vivo is about one linker histone molecule per nucleosome [19], it is variable and appears related to the nucleosome repeat length (NRL). For example, neuronal chromatin has a NRL of $162 \mathrm{bp}$ and 0.45 molecules of $\mathrm{H} 1$ per nucleosome, whereas in glial cells the NRL is $201 \mathrm{bp}$ and the stoichiometry is 1.04 [53] and in natural retinal maturation the NRL gradually increases from 190 to $206 \mathrm{bp}$ as the amount of $\mathrm{H} 1$ increases by 23\% [54]. Knock-outs of individual H1 subtypes conducted in mouse ES cells led finally to only $\sim 50 \%$ of wt levels of $\mathrm{H} 1$ and it was observed that the NRL fell in proportion to the amount of $\mathrm{H} 1$ lost $[55,56]$. In fact, a plot of all available in vivo data showed a good linear relationship from yeast ( $\mathrm{NRL}=165 \mathrm{bp}$, no conventional $\mathrm{H} 1$ ) to chicken erythrocyte nuclei (NRL=211 bp, $1.3 \mathrm{H} 1 \mathrm{~s} /$ nucleosome) [57].

The role of the $\mathrm{H} 1$ molecule in this relationship seems to be the maintenance of electrostatic charge homeostasis, outcome being that its binding keeps adjacent nucleosomes apart and achieves their regular spacing. In the knock-out experiments 
$[55,56]$ the reduced amounts of the prime agent of chromosome condensation that led to artificially lowered NRL values might be expected to be accompanied by substantial increases in transcription - but that was not found to be the case [56]. This could be the consequence of $\mathrm{H} 1$ depletion being concentrated in inactive regions of the genome but, strikingly, in ESCs (having a quite low average NRL of $185 \mathrm{bp}$ and only about 0.5 molecules of $\mathrm{H} 1$ per nucleosome) there was no clear distinction between observed NRLs for active genes and inactive heterochromatin [56]. There is therefore no general link between the capacity for transcription and the amount of H1 present.

There is however a link between local levels of $\mathrm{H} 1$ and actual/potential transcriptional activity [58]. Experiments to map the distribution of linker histones across the genome show reductions in the concentration of $\mathrm{H} 1$ at DNaseI hypersensitive sites (DHS), e.g. at gene promoters and enhancers. Clear-cut results were obtained in Drosophila and human cells using the DAM-methylase mapping protocol. Although not yielding particularly high resolution, very clear 'dips' of low H1 occupancy were noted at the TSS of active genes and at multiple cis-regulatory sites $[59,60]$. Such reductions are not simply a consequence of nucleosome depletion, as no concomitant loss of core histone was seen. Similar 'dips' were noted when antibodies against $\mathrm{H} 1$ subtypes and variants were used with several chicken cell types [61]. Such observations indicate that H1 needs to be displaced from cisregulatory elements to facilitate their function. Along the body of active genes, however, substantial amounts of H1 remain, though at depleted levels [62].

\section{H1 and chromatin remodelling}

The important question is then: how is access gained to H1-containing compacted ('closed') chromatin in order to initiate transcription? The answer lies in a subset of transcription factors - pioneer transcription factors (PTFs) - that can access their target sites on wrapped, nucleosomal DNA in compacted chromatin, rather than requiring exposed targets on inter-nucleosomal DNA, i.e. the binding of PTFs is not inhibited by the presence of H1. Striking early examples of PTFs were the factors 
HNF-3 (FoxA) and GATA-4 that were shown to target albumin gene enhancer elements in reconstituted H1-compacted fibres, leading to opening of the chromatin in the absence of remodelers [63]. An opening mechanism was proposed in which the C-terminal domain of HNF-3 binds to core histone tails, preventing them from stabilising the chromatin higher order structure. Hormone receptors were early recognised as binding to their targets on nucleosomal DNA [64] and the PTF list is now much extended, in particular to include the pluripotency-inducing factors Oct4, Sox2 and Klf4 [65]. These represent a broad range of DBD folds, so there is no universal mechanism by which PTFs open up H1-compacted chromatin but it is clear that binding of PTFs is followed by recruitment of acetyltransferases (HATs), remodeling complexes and other key factors that open up and re-shuffle the chromatin, leading to local loss of $\mathrm{H} 1$ [66].

Some chromatin remodelling steps have been individually investigated: for example, it has been shown that H1-containing nucleosomes and more importantly, H1-containing nucleosomal arrays can be remodelled by ACF (but not by CHD1) [67]. The same is true for the SWI/SNF complex but only if the H1 is phosphorylated [68]. The ability of ACF to remodel H1-containing chromatin elements certainly depends on the type of linker histone: the xenopus oocyte-specific B4 histone fails to inhibit accessibility of the linker DNA and thus permits remodelling. This is in contrast to the somatic histone H1A which is able to block remodelling [69] - at least for a dinucleosomal substrate - a difference that could be a direct consequence of their very different CTDs.

Displacement of $\mathrm{H} 1$ can be regarded as a prerequisite for transcription $[63,66]$ and reciprocal binding of PARP1 following loss of $\mathrm{H} 1$ at promoters has been described [70], as has the recruitment of the variant histone H2A.Z [71-73] following binding of a PTF. An active promoter is therefore envisaged as lacking H1, with several other factors having gained access.

A more recent in vitro-assembled H1-containing transcriptional system [74] involved chromatin assembled on a $5 \mathrm{~kb}$ plasmid containing $540 \mathrm{bp}$ from the rat PEPCK promoter, which can be induced by addition of retinoic acid (RA) in 
consequence of incorporating two RAREs. Reconstitution used unacetylated core histones and $\mathrm{H} 1$ in the presence of chaperones and the state of compaction of the template was monitored by EM, showing condensed fibres of 26-28 nm diameter. In this state the template was refractory to transcription, however following treatment with the PTF RAR/RXR, addition of the remodeler SWI/SNF and the HAT p300 was then sufficient to displace nucleosomes, release the $\mathrm{H} 1$ and permit binding of NF1 with subsequent transcription. The sequence of these in vitro activating events followed the main features of the regulated expression of the PEPCK gene in vivo.

Multiple subtypes of H1 Whilst the variant linker histones such as H5, H1.0 and B4 have been the subject of multiple studies, the function of the replication-dependent subtypes that make up the bulk of the H1 presents a more difficult problem, not least because their primary structures vary little [75]. Despite the fact that linker histone subtypes appear to act as redundant proteins in knock-out mice [55], suggesting no functional distinction between such subtypes, different tissues are nevertheless characterized by the action of specific linker histone subtypes and changes in subtype expression are observed during development [76-79].

One approach to this question has been the use of subtype specific antibodies in ChIP studies - with rather mixed results. In human cells H1.5 (not one of the most abundant RD subtypes) binds in blocks of enrichment to certain genic and intergenic regions, leading to the binding of Sirt1 and the dimethylation of H3K9, suppressing their activity [77]. Using knock-in FLAG-tagged H1 species in ChIPs with mouse ESC, blocks of H1c and H1d enrichment (abundant RD subtypes) were seen at satellite DNA sequences and their depletion noted at active promoters [80].

Some functional distinctions between the RD subtypes have been documented in gene expression studies [81] that frequently show recruitment of specific $\mathrm{H} 1$ subtypes to genes subject to repression in consequence of the interaction of the subtype with an established transcription factor (TF). In one case a pioneer transcription factor, FoxP3, interacts with human H1.5 to repress IL-2 expression in 
T-cells [82]. Other examples are mouse H1b interacting with Msx1 to negatively regulate MyoD expression in myoblasts [83] and human H1.2 interacting with p53 to direct H1.5 to genes targeted by p53 in transfected HeLa cells [84].

As functional distinctions between different $\mathrm{H} 1$ subtypes have been clearly made, the challenge now is to refine these and determine the molecular mechanisms whereby these distinctions are brought about. One possibility is that variations in the primary structure of the common RD subtypes give rise to differences in their epigenetic modifications. A striking example of this is the methylation of K26 in human H1.4 that leads to recruitment of the repressor HP1, provided the adjacent S27 is not phosphorylated. The K26/S27 pair (representing a phospho-switch) is unique to the H1.4 subtype in this region of the NTD [85]. The sequence variability and frequent modification of this domain make it the primary candidate for the regulatory element of the linker histones.

\section{REFERENCES}

1) K. Luger, A.W. Mader, R.K. Richmond, D.F. Sargent, T.J. Richmond, Crystal structure of the nucleosome core particle at $2.8 \AA$ r resolution, Nature 389 (1997) 251-260.

2) M.A. Lever, J.P. Th'ng, X. Sun, M.J. Hendzel, Rapid exchange of histone H1.1 on chromatin in living cells, Nature 408 (2000) 873-876.

3) T. Misteli, A. Gunjan, R. Hock, M. Bustin, D.T. Brown, Dynamic binding of histone H1 to chromatin in living cells, Nature 408 (2000) 877-881.

4) J. Allan, P.G. Hartman, C. Crane-Robinson, F.X. Aviles, The structure of histone H1 and its location in chromatin, Nature 288 (1980) 675-679.

5) V. Ramakrishnan, J.T. Finch, V. Graziano, P.L. Lee, R.M. Sweet, Crystal structure of the globular domain of histone H5 and its implications for nucleosome binding, Nature 362 (1993) 219-224.

6) J. Allan, T. Mitchell, N. Harborne, L. Bohm, C. Crane-Robinson, Roles of H1 domains in determining higher order chromatin structure and H1 location. J. Mol. Biol. 187 (1986) 591-601.

7) M.J. Hendzel, M.A. Lever, E. Crawford, J.P. Th'ng, The C-terminal domain is the primary determinant of histone H1 binding to chromatin in vivo. J. Biol. Chem. 279 (2004) 20028-20034.

8) X. Lu, B. Hamkalo, M.H. Parseghian, J.C. Hansen, Chromatin condensing functions of the linker histone $\mathrm{C}$-terminal domain are mediated by specific amino acid composition and intrinsic protein disorder, Biochemistry 48 (2009) 164-172.

9) A. Luque, R. Collepardo-Guevara, S. Grigoryev, T. Schlick, Dynamic condensation of linker histone C-terminal domain regulates chromatin structure, Nucleic Acids Research 42 (2014) 7553-7560.

10) H. Fang, D.J. Clark, J.J. Hayes, DNA and nucleosomes direct distinct folding of a linker histone H1 
C-terminal domain, Nucleic Acids Research 40 (2012) 1475-1484.

11) R. Lopez, B. Sarg, H. Lindner, S. Bartolome, I. Ponte, P. Suau, A. Roque, Linker histone partial phosphorylation: effects on secondary structure and chromatin condensation, Nucleic Acids Research 43 (2015) 4463-4476.

12) W. Albig, B. Drabent, J. Kunz, M. Kalff-Suske, K.-H. Grzeschik, D. Doenecke, All Known Human H1 Histone Genes Except the H1 ${ }^{0}$ Gene Are Clustered on Chromosome 6, Genomics 16, (1993) 649-654.

13) L. Hereford, K. Fahrner, J. Woolford Jr., M. Rosbash, D.B. Kaback, Isolation of yeast histone genes H2A and H2B. Cell 18 (1979) 1261-1271.

14) M.M. Smith, O.S. Andresson, DNA sequences of yeast $\mathrm{H} 3$ and $\mathrm{H} 4$ histone genes from two non-allelic gene sets encode identical H3 and H4 proteins. J. Mol. Biol. 169, (1983) 663-690.

15) E.M. Bradbury, R.J. Inglis, H.R. Matthews, T.A. Langan, Molecular basis of control of mitotic cell division in eukaryotes, Nature 249 (1974) 553-556.

16) C. Giri, M. West, M. Smulson, Nuclear protein modification and chromatin substructure. 1.

Differential poly(adenosine diphosphate) ribosylation of chromosomal proteins in nuclei versus isolated nucleosomes. Biochemistry 17 (1978) 3495-3500.

17) J.R. Wisniewski, A. Zougman, S. Kruger, M. Mann, Mass spectrometric mapping of linker histone $\mathrm{H} 1$ variants reveals multiple acetylations, methylations, and phosphorylation as well as differences between cell culture and tissue, Mol. Cell. Proteomics 6 (2007) 72-87.

18) M.A. Christophorou, G. Castelo-Branco, R.P. Halley-Stott, C.S. Oliveira, R. Loos, A. Radzisheuskaya, K.A. Mowen, P. Bertone, J.C. Silva, M. Zernicka-Goetz, M.L. Nelson, J.B. Gurdon, T. Kouzarides, Citrullination regulates pluripotency and histone H1 binding to chromatin. Nature 507 (2014) 104-108.

19) D.L. Bates, J.O. Thomas, Histones H1 and H5: one or two molecules per nucleosome, Nucleic Acids Research 25 (1981) 5883-5894.

20) D.Z. Staynov, C. Crane-Robinson, Footprinting of linker histones H5 and H1 on the nucleosome. EMBO J 7 (1988) 3685-3691.

21) D. Pruss, B. Bartholomew, J. Persinger, J.J. Hayes, G. Arents, E.N. Moudrianakis, A.P. Wolffe, An asymmetric model for the nucleosome: A binding site for linker histones inside the DNA gyres, Science 274 (1996) 614-617.

22) L. Fan, V.A. Roberts, Complex of linker histone H5 with the nucleosome and its implications for chromatin packing. Proc Natl Acad Sci USA 103 (2006) 8384-8389.

23) D.T. Brown, T. Izard, T. Misteli, Mapping the interaction surface of linker histone H1(0) with the nucleosome of native chromatin in vivo. Nat. Struct. Mol. Biol. 13 (2006) 250-255.

24) F.A. Goytisolo, S.E. Gerchman, X. Yu, C. Rees, V. Graziano, V. Ramakrishnan, J.O. Thomas, Identification of two DNA-binding sites on the globular domain of histone H5. EMBO J. 15 (1996) 34213429.

25) M.M. Duggan, J.O. Thomas, Two DNA binding sites on the globular domain of histone H5 are required for binding to both bulk and $5 \mathrm{~S}$ reconstituted nucleosomes. J. Mol. Biol. 304 (2000) 21-33.

26) Y.-B. Zhou, S.E. Gerchman, V. Ramakrishnan, A. Travers, S. Muyldermans, Position and orientation 
of the globular domain of linker histone H5 on the nucleosome. Nature 395 (1998) 402-405.

27) V. Graziano, S.E. Gerchman, D.K. Schneider, V. Ramakrishnan, Histone H1 is located in the interior of the chromatin 30-nm filament. Nature 368 (1994) 351-354.

28) J.O. Thomas, C.M. Wilson, Selective radiolabelling and identification of a strong nucleosome binding site on the globular domain of histone H5, EMBO J 5 (1986) 3531-3537.

29) C. Crane-Robinson, O.B. Ptitsyn, Binding of the globular domain of linker histones H5/H1 to the nucleosome: a hypothesis, Protein Engineering 2 (1989) 577-582.

30) F. Cui, V.B. Zhurkin, Distinctive sequence patterns in metazoan and yeast nucleosomes: Implications for linker histone binding to AT-rich and methylated DNA, Nucleic Acids Research 37 (2009) 28182829.

31) S.H. Syed, D. Goutte-Gattat, N. Becker, S. Meyer, M.S. Shukla, J.J. Hayes, R. Everaers, D. Angelov, J. Bednar, S. Dimitrov, Single-base resolution mapping of H1-nucleosome interactions and 3D organization of the nucleosome. Proc Natl Acad Sci USA 107 (2010) 9620-9625.

32) F. Song, P. Chen, D. Sun, M. Wang, L. Dong, D. Liang, R.-M. Xu, P. Zhu, G. Li, Cryo-EM Study of the Chromatin Fiber Reveals a Double Helix Twisted by Tetranucleosomal Units, Science 344 (2015) $376-380$.

33) T. Schalch, S. Duda, D.F. Sargent, T.J. Richmond, X-ray structure of a tetra-nucleosome and its implications for the chromatin fibre. Nature 436 (2005) 138-141.

34) B.-R. Zhou, J. Jiang, H. Feng, R. Ghirlando, T.S. Xiao, Y. Bai, Structural Mechanisms of Nucleosome Recognition by Linker Histones. Mol. Cell 59 (2015) 1-11,

35) B.-R. Zhou, H. Feng, H. Kato, L. Dai, Y. Yang, Y. Zhou, Y. Bai, Structural insights into the histone H1-nucleosome complex. Proc Natl Acad Sci USA 110 (2013) 19390-19395.

36) J.T. Finch, A. Klug, Solenoidal model for superstructure in chromatin. Proc. Natl. Acad. Sci. U. S. A. 73 (1976) 1897-1901.

37) F. Thoma, T. Koller, A. Klug, Involvement of histone H1 in the organization of the nucleosome and of the salt-dependent superstructures of chromatin. J. Cell. Biol. 83 (1979) 403-427.

38) S.E. Gerchman, V. Ramakrishnan, Chromatin higher-order structure studied by neutron scattering and scanning transmission electron microscopy. Proc. Natl. Acad. Sci. U. S. A. 84 (1987) 7802-7806.

39) J. Bednar, R.A. Horowitz, S.A. Grigoryev, L.M. Carruthers, J.C. Hansen, A.J. Koster, C.L. Woodcock, Nucleosomes, linker DNA, and linker histone form a unique structural motif that directs the higher-order folding and compaction of chromatin. Proc. Natl. Acad. Sci. U. S. A. 95 (1998) 1417314178.

40) P.M. Schwarz, A. Felthauser, T.M. Fletcher, J.C. Hansen, Reversible oligonucleosome self association: dependence on divalent cations and core histone tail domains. Biochemistry 35 (1996) 40094015.

41) I. Nazarov, Personal Communication.

42) D.Z. Staynov, Possible nucleosome arrangements in the higher-order structure of chromatin. Int. J. Biol. Macromol. 5 (1983) 3-9.

43) P.T. Lowary, J. Widom, New DNA sequence rules for high affinity binding to histone octamer and 
sequence-directed nucleosome positioning. J. Mol. Biol. 276 (1998) 19-42.

44) J.R. Daban, A. Bermudez, Interdigitated solenoid model for compact chromatin fibers. Biochemistry 37 (1998) 4299-4304.

45) P.J. Robinson, L. Fairall, V.A. Huynh, D. Rhodes, EM measurements define the dimen sions of the “'30-nm' chromatin fiber: Evidence for a compact, interdigitated structure. Proc. Natl. Acad. Sci. USA 103 (2006) 6506-6511.

46) A. Routh, S. Sandin, D. Rhodes, Nucleosome repeat length and linker histone stoichi ometry determine chromatin fiber structure. Proc Natl Acad Sci USA 105 (2008) 8872-8877.

47) M.P. Scheffer, M. Eltsov, A.S. Frangakis, Evidence for short-range helical order in the 30-nm chromatin fibers of erythrocyte nuclei. Proc. Natl. Acad. Sci. USA 108 (2011) 16992-16997.

48) R. Ghirlando, G. Felsenfeld, Hydrodynamic studies on defined heterochromatin fragments support a 30-nm fiber having six nucleosomes per turn. J. Mol. Biol. 376 (2008) 1417-1425.

49) M. Eltsov, K.M. Maclellan, K. Maeshima, A.S. Frangakis, J. Dubochet, Analysis of cryo-electron microscopy images does not support the existence of 30-nm chromatin fibers in mitotic chromosomes in situ. Proc. Natl. Acad. Sci. USA 105 (2008) 19732-19737.

50) Y. Nishino, M. Eltsov, Y. Joti, K. Ito, H. Takata, Y. Takahashi, S. Hihara, A.S. Frangakis, N. Imamoto, T. Ishikawa, K. Maeshima, Human mitotic chromosomes consist predominantly of irregularly folded nucleosome fibers without a 30-nm chromatin structure. EMBO J. 31 (2012) 1644-1653.

51) E. Fussner, M. Strauss, U. Djuric, R. Li, K. Ahmed, M. Hart, J. Ellis, D.P. Bazett-Jones, Open and closed domains in the mouse genome are configured as 10-nm chromatin fibres. EMBO Rep. 13 (2012) 992-996.

52) E. Meshorer, D. Yellajoshula, E. George, P.J. Scambler, D.T. Brown, T. Misteli, Hyperdynamic plasticity of chromatin proteins in pluripotent embryonic stem cells. Dev. Cell 10 (2006) 105-116.

53) E.C. Pearson, D.L. Bates, T.D. Prospero, J.O. Thomas, Neuronal nuclei and glial nuclei from mammalian cerebral cortex, Nucleosome repeat lengths, DNA contents and H1 contents. Eur. J. Biochem. 144 (1984) 353-360.

54) E.Y. Popova, S.A. Grigoryev, Y. Fan, A.I. Skoultchi, S.S. Zhang, C.J. Barnstable, Developmentally Regulated Linker Histone H1c Promotes Heterochromatin Condensation and Mediates Structural Integrity of Rod Photoreceptors in Mouse Retina. J. Biol. Chem. 288 (2013) 17895-17907.

55) Y. Fan, T. Nikitina, E.M. Morin-Kensicki, J. Zhao, T.R. Magnuson, C.L. Woodcock, A.I. Skoultchi, (2003) H1 linker histones are essential for mouse development and affect nucleosome spacing in vivo, Mol. Cell. Biol. 23 (2003) 4559-4572.

56) Y. Fan, T. Nikitina, J. Zhao, T.J. Fleury, R. Bhattacharyya, E.E. Bouhassira, A. Stein, C.L. Woodcock, A.I. Skoultchi, Depletion of histone H1 in mammals alters global chromatin structure but causes specific changes in gene regulation. Cell 123 (2005) 1199-1212.

57) C.L. Woodcock, A.I. Skoultchi, Y. Fan, Role of linker histone in chromatin structure and function: H1 stoichiometry and nucleosome repeat length. Chromosome Research 14 (2006) 17-25.

58) S. Kadota, K. Nagata, Silencing of IFN-stimulated gene transcription is regulated by histone $\mathrm{H} 1$ and its chaperone TAF-I. Nucleic Acids Research 42 (2014) 7642-7653.

59) U. Braunschweig, G.J. Hogan, L. Pagie, B. van Steensel, Histone H1 binding is inhibited by histone 
variant H3.3. EMBO J. 28 (2009) 3635-3645.

60) A. Izzo, K. Kamieniarz-Gdula, F. Ramirez, N. Noureen, J. Kind, T. Manke, B. van Steensel, R. Schneider, The Genomic Landscape of the Somatic Linker Histone Subtypes H1.1 to H1.5 in Human Cells. Cell Reports 3 (2013) 2142-2154.

61) A.F. Trollope, N. Sapojnikova, A.W. Thorne, C. Crane-Robinson, F.A. Myers, Linker histone subtypes are not generalized gene repressors. Biochim. Biophys. Acta 1799 (2010) 642-652.

62) L. Millan-Arino, A. Islam, A. Izquierdo-Bouldstridge, R. Mayor, J.-M. Terme, N. Luque, M. Sancho, N. Lopez-Bigas, A. Jordan, Mapping of six somatic linker histone H1 variants in human breast cancer cells uncovers specific features of H1.2. Nucleic Acids Research 42 (2014) 4474-4493.

63) L.A. Cirillo, F.R. Lin, I. Cuesta, D. Friedman, M. Jarnik, K.S. Zaret, Opening of compacted chromatin by early developmental transcription factors HNF3 (FoxA) and GATA-4, Mol. Cell 9 (2002) 279-289.

64) T. Archer, M.G. Cordingly, R.G. Wolford, G.L. Hager, Transcription Factor Access Is Mediated by Accurately Positioned Nucleosomes on the Mouse Mammary Tumor Virus Promoter. Mol. Cell. Biol. 11 (1991) 688-698.

65) A. Soufi, G. Donahue, K.S. Zaret, Facilitators and Impediments of the Pluripotency Reprogramming Factors' Initial Engagement with the Genome. Cell 151 (2012) 994-1004.

66) G.P. Vicent, A.S. Nacht, J. Font-Mateu, G. Castellano, L. Gaveglia, C. Ballare, M. Beato, Four enzymes cooperate to displace histone $\mathrm{H} 1$ during the first minute of hormonal gene activation. Genes Dev. 25 (2011) 845-862.

67) V.K. Maier, M. Chioda, D. Rhodes, P.B. Becker, Acf catalyses chromatosome movements in chromatin fibres. EMBO J. 27 (2008) 817-826.

68) P.J. Horn, L.M. Carruthers, C. Logie, D.A. Hill, M.J. Solomon, P.A. Wade, A.N. Imbalzano, J.C. Hansen, C.L. Peterson, Phosphorylation of linker histones regulates ATP-dependent chromatin remodeling enzymes. Nat. Struct. Biol. 9 (2002) 263-267.

69) H. Saeki, K. Ohsumi, H. Aihara, T. Ito, S. Hirose, K. Ura, Y. Kaneda, Linker histone variants control chromatin dynamics during early embryogenesis. Proc. Natl. Acad. Sci. USA 102 (2005) 5697-5702.

70) R. Krishnakumar, M.J. Gamble, K.M. Frizzell, J.G. Berrocal, M. Kininis, W.L. Kraus, Reciprocal binding of PARP-1 and histone H1 at promoters specifies transcriptional outcomes. Science 319 (2008) 819-821.

71) Z. Li, P. Gadue, K. Chen, Y. Jiao, G. Tuteja, J. Schug, W. Li, K.H. Kaestner, FoxA2 and H2A.Z Mediate Nucleosome Depletion during Embryonic Stem Cell Differentiation. Cell 151 (2012) 16081616.

72) J.S. Menet, S. Pescatore, M. Rosbash, CLOCK:BMAL1 is a pioneer-like transcription factor. Genes Dev. 28 (2014) 8-13.

73) D.L. Updike, S.E. Mango, Temporal Regulation of Foregut Development by HTZ-1/H2A.Z and PHA-4/FoxA. PLoS Genetics 2 (2006) e161.

74) G. Li, R. Margueron, G. Hu, D. Stokes, Y.-H. Wang, D. Reinberg, Highly Compacted Chromatin Formed In Vitro Reflects the Dynamics of Transcription Activation In Vivo. Mol. Cell 38 (2010) 4153. 
75) M.H. Parseghian, B.A. Hamkalo, A compendium of the histone H1 family of somatic subtypes: an elusive cast of characters and their characteristics. Biochem. Cell. Biol. 79 (2001) 289-304.

76) J. Clausell, N. Happel, T. K. Hale, D. Doenecke, M. Beato, Histone H1 subtypes differentially modulate chromatin condensation without preventing ATP-dependent remodeling by SWI/SNF or NURF. PLoS One 4 (2009) e0007243.

77) J.-Y. Li, M. Patterson, H.K.A. Mikkola, W.E. Lowry, S.K. Kurdistani, Dynamic Distribution of Linker Histone H1.5 in Cellular Differentiation. PLoS Genetics 8 (2012) e1002879.

78) K. Kamieniarz, A. Izzo, M. Dundr, P. Tropberger, L. Ozretic, J. Kirfel, E. Scheer, P. Tropel, J.R. Wisniewski, L. Tora, S. Viville, R. Buettner, R. Schneider, A dual role of linker histone H1.4 Lys-34 acetylation in transcriptional activation. Genes Dev. 26 (2012) 797- 802.

79) J.-M. Terme, B. Sese, L. Millan-Arino, R. Mayor, J.C. Izpisua Belmonte, M.J. Barrero, A. Jordan, Histone H1 Variants Are Differentially Expressed and Incorporated into Chromatin during Differentiation and Reprogramming to Pluripotency. J. Biol. Chem. 286 (2011) 35347-35357.

80) K. Cao, N. Lailler, Y. Zhang, A. Kumar, K. Uppal, Z. Liu, E.K. Lee, H. Wu, M. Medrzycki, C. Pan, P.Y. Ho, G.P. Cooper Jr, X. Dong, C. Bock, E.E. Bouhassira, Y. Fan, High-resolution mapping of H1 linker histone variants in embryonic stem cells. PLoS Genet. 9 (2013) e1003417.

81) R. Alami, Y. Fan, S. Pack, T.M. Sonbuchner, A. Besse, Q. Lin, J.M. Greally, A.I. Skoultchi, E.E. Bouhassira, Mammalian linker-histone subtypes differentially affect gene expression in vivo. Proc. Natl. Acad. Sci. USA 100 (2003) 5920-5925.

82) S.L. Mackey-Cushman, J. Gao, D.A. Holmes, J.-I. Nunoya, R. Wang, D. Unutmaz, L. Su, FoxP3 interacts with linker histone H1.5 to modulate gene expression and program Treg cell activity. Genes \& Immunity 12 (2011) 559-567.

83) H. Lee, R. Habas, C. Abate-Shen, Msx 1 cooperates with histone H1b for inhibition of transcription and myogenesis. Science 304 (2004) 1675-1678.

84) K. Kim, J. Choi, K. Heo, H. Kim, D. Levens, K. Kohno, E.M. Johnson, H.W. Brock, W. An, Isolation and Characterization of a Novel H1.2 Complex That Acts as a Repressor of p53-mediated Transcription. J. Biol. Chem. 283 (2008) 9113-9126.

85) S. Daujat, U. Zeissler, T. Waldmann, N. Happel, R. Schneider, HP1 binds specifically to K26 methylated histone H1.4, whereas simultaneous S27 phosphorylation blocks HP1 binding. J. Biol. Chem. 280 (2005) 38090-38095. 